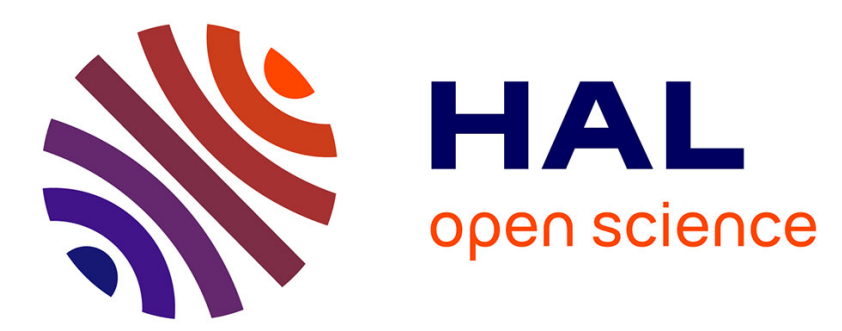

\title{
Localisation, identité et origine du Javā de Jayavarman II
}

\author{
Michel Ferlus
}

\section{To cite this version:}

Michel Ferlus. Localisation, identité et origine du Javā de Jayavarman II. Aséanie, Sciences humaines en Asie du Sud-Est, 2010, 26, pp.65-81. halshs-01571570

\section{HAL Id: halshs-01571570 \\ https://shs.hal.science/halshs-01571570}

Submitted on 2 Aug 2017

HAL is a multi-disciplinary open access archive for the deposit and dissemination of scientific research documents, whether they are published or not. The documents may come from teaching and research institutions in France or abroad, or from public or private research centers.
L'archive ouverte pluridisciplinaire HAL, est destinée au dépôt et à la diffusion de documents scientifiques de niveau recherche, publiés ou non, émanant des établissements d'enseignement et de recherche français ou étrangers, des laboratoires publics ou privés.

\section{(1)(1) $\$(0)$}

Distributed under a Creative Commons Attribution - NonCommercial - ShareAlikel 4.0 


\title{
Localisation, identité et origine du Javā de Jayavarman II
}

\author{
Michel Ferlus
}

Cet article a été publié dans la revue Aséanie, $\mathrm{n}^{\circ}$ 26, décembre 2010, pp. 65-81.

\section{à Tatsuo Hoshino}

1. Selon George Cœdès, Jayavarman II fondateur de la royauté angkorienne, aurait libéré le Cambodge de la suzeraineté de Javā vers $802^{1}$. On n'a pas trouvé de stèle de ce souverain mais les épisodes de son avènement et de son règne seront relatés deux siècles plus tard dans la célèbre inscription de Sdok Kak Thom (K.235, XIe siècle). ${ }^{2}$ Le texte nous dit : "Sa majesté vint de Javā pour régner dans la cité d'Indrapura »... Ensuite, le roi alla s'installer dans la plaine au nord du Grand Lac accompagné d'un savant brahmane comme chapelain. Après avoir régné à Hariharalāya puis fondé la cité d'Amarendrapura, le roi installa sa capitale sur le mont Mahendra (Phnom Kulen) et fit venir un brahmane savant dans la science magique afin d'assurer le rituel du Devarāja et l'enseigner au chapelain, "pour que le pays des Kambujas ne fût plus dépendant de Javā, et qu'il n'y eût plus qu'un seul souverain qui fût chakravartin », c'est à dire monarque universel.

Sans prendre trop de risques, il est possible d'imaginer la succession des événements rapportés dans l'inscription de Sdok Kak Thom.. Le futur Jayavarman II quitte un pays nommé Javā (rébellion contre un pouvoir étranger!), s'installe (par conquête) à Indrapura situé quelque part au sud-est du Grand Lac, puis se déplace dans la plaine de la future Angkor (il agrandit son domaine) et se fait proclamer monarque universel sur le mont Mahendra (rejet de la suzeraineté de Javā et unification des Kambujas). Ce raccourci s'inspire librement des conclusions de Claude Jacques (1972: 219-20) sur la carrière de ce grand conquérant unificateur que fut Jayavarman II. Si le cadre historique est bien compris dans ses grandes lignes, la localisation du Javā reste toujours le grand problème non résolu.

\footnotetext{
${ }^{1}$ Voir Les états hindouisés d'Indochine et d'Indonésie, chap. VII, §.1, 1948 pp. 166-178, ou 1989 pp. 183-194.

${ }^{2}$ Cette stèle découverte en Thaïlande non loin de la frontière du Cambodge est aujourd'hui déposée au Musée National de Bangkok. Elle a fait l'objet de nombreuses publications : Aymonier (1901), Finot (1915), Cœdès \& Dupont (1943-46) et, plus récemment, Sak Humphry (2005).
} 
Avant tout épigraphiste, George Cœdès (1948) a tout naturellement identifié le Javā de Jayavarman II avec le Java indonésien, opinion renforcée par la renommée de son ouvrage Les états hindouisés qui influencera durablement les spécialistes de l'Asie du Sud-Est. Il faut cependant reconnaître que le problème de l'origine du Javā, qui n'hypothèque en rien la suite des événements, ne semble pas faire préoccupation dans l'œuvre monumentale de ce grand savant. En dehors de Pierre Dupont, co-auteur avec George Cœdès (1943-46), et de Wolters (1973: 21, note 8) la plupart resteront réservés sur l'identification du Javā de Jayavarman II avec le Java indonésien.

Il était pourtant bien connu, depuis au moins le début du XXe siècle, que l'île nommée actuellement Java s'appelait autrefois Yavadvīpa "l'île de Yava". Louis Finot l'a affirmé par deux fois : d'abord en commentant la stèle cham de Po-sah il écrit (1903: 641, note 1) «On voit par là que Javā était un royaume parfaitement distinct de l'île de Java, laquelle était appelée Yava, Yavadvīpa », puis quelques années plus tard (1915: 57) « Ce roi était venu de Javā pour régner au Cambodge. On ignore quel pays ce nom désigne ici; ni Java, ni Louang-Prabang ne semblent historiquement possibles ». Ces remarques étaient forcément connues de Cœdès (1948: 92-93 / 1989: 104-105) tandis qu'il écrivait «L'île de Java est probablement citée dans le Rāmāyaṇa (Yavadvīpa) et dans Ptolémée (Iabadiou) », mais curieusement il n'en a pas tenu compte. Jan Gonda (1973: 348-49) rapporte que Ptolémée attribue à Iabadiou (Yavadvipa) le sens de "île de l'orge" (Skt. yava "orge", dvipa "île") ou de toute autre céréale comestible, millet ou riz. Devant l'improbabilité de ces significations, Gonda pense que Yava pouvait tout simplement désigner les autochtones. Le changement de l'ancien nom Yava en Java (d'Indonésie) est conforme à la loi phonétique qui veut que skt. $y$ passe régulièrement à $j$ en prakrit (Renou \& Filliozat 1985: 78-79).

Michael Vickery (1998: 28-29) situe le point de départ de Jayavarman II dans l'est du Cambodge, à Vyādhapura, aux limites du Champa, et propose d'identifier le Javā avec le Champa (dans le sud du Vietnam actuel).

Pour Bruno Dagens (2003: 27) « ... ce pays n'est pas nécessairement comme on l'a cru longtemps l'île de Java; il peut aussi bien s'agir du Champa ou d'une autre région proche du Cambodge $»$.

Claude Jacques (2005: 21-22) rappelle bien que l'île de Java était à ce momentlà nommée Yava/ Yavadvīpa mais pense néanmoins que Javā doit représenter une 'terre de Java' localisée dans la péninsule malaise.

Une nouvelle impulsion au problème de l'identification et de la localisation du Javā devait être donnée par l'historien Tatsuo Hoshino (1986: 42-46) dont les idées sont développées dans ce qui suit.

\section{L'hypothèse de Tatsuo Hoshino}

Le débat sur l'identification du Javā est resté longtemps confiné au cercle des khmérisants, quelle que soit leur opinion sur le sujet; influencés par l'autorité scientifique de George Cœdès, ils ont négligé les sources chinoises autres que celles exploitées par quelques sinologues. L'originalité de Tatsuo Hoshino a justement été de savoir utiliser des données inédites des textes chinois non traduits dans des langues 
occidentales et de les confronter aux données épigraphiques de l'Asie du Sud-Est (Hoshino 1986 et 2002).

La Nouvelle histoire des T'ang (618-907) mentionne un pays nommé Shepo/Chö-po (shépó 闍婆, ou shèpó 社婆) vassal des Pyu (Pyū), ou P'iao, qui formaient un Etat sur l'Irrawadi dans le centre de la Birmanie. Dès le début du XXe siècle, Pelliot (1904: 265-266) avait reconnu sous la graphie de Shepo le nom de Javā qu'il avait identifié, erreur n'est pas coutume, au Java indonésien alors que dans le même temps Finot (1903: 641, note 1) rejetait cette localisation. Il faut toutefois reconnaître que le problème de l'ambivalence des dénominations Javā/Java est également présent dans les sources chinoises : à côté du Shepo continental de l'époque T'ang, l'Histoire des Yuan nous révèle un autre Shepo (Shépó 蛇婆) qui désignait sans conteste le Java indonésien (Kurz 2006: 5), avec cependant la différence que ces deux désignations homonymes n'étaient pas confondues par les Chinois.

Une inscription mône du XIe siècle atteste l'existence d'une population nommée ja 'ba' que Harry Shorto (1971: 117) glose "Native of the region of northern Laos". Or, la tradition lao situe justement à Louang Phrabang le Muong Swa (écrit jvā २อๆ, prononcé swa:), premier royaume lao. Le nom de ce pays apparaît également dans la stèle maîtresse de l'épigraphie thaï, la célèbre inscription de Ramkhamhèng ${ }^{4}$ qui a dû être gravée au tout début du XIVe siècle dans la région de Sukhothay.

Hoshino pense que les termes shépó 闍婆 en chinois, ja'ḅa' en môn et jvā en lao et en thaï ne sont que des variantes de javā en khmer, c'est à dire du Javā dont Jayavarman II a rejeté la suzeraineté en se proclamant souverain universel sur le mont Mahendra en 802. L'auteur propose d'identifier ce Javā avec le Zhenla de terre (partie nord du Cambodge au VIIIe siècle) de capitale Wen Dan. Cet état se serait étendu sur le Moyen et Nord Laos au VIIIe siècle, sa dernière ambassade à la capitale des T'ang date de 799. Hoshino continue (1986: 43) : "Après cette date, un silence complet du côté des Chinois règne sur ce pays, ce qui nous semble une coïncidence curieuse avec la déclaration de l'indépendance khmère à l'égard du Javā ». Il semblerait qu'un Javā se soit perpétué dans le Nord-Laos pendant quelques siècles jusqu'à la conquête de Ramkhamhèng.

Rappelons que le nom de Zhenla/Tchen-la apparaît vers 550 dans les sources chinoises comme successeur du Funan/Fou-nan; apparemment simple changement onomastique qui n'implique aucun bouleversement particulier. Durant le VIIIe, les Chinois traitent le Zhenla comme deux pays distincts, le Zhenla de terre et le Zhenla d'eau. Ils entretiennent des rapports avec les deux ce qui montre bien qu'ils formaient deux entités qui, comme on le sait, seront réunifiés en l'an 802 par Jayavarman II.

Telle est la situation actuelle : dans l'ensemble, les spécialistes n'admettent pas l'identification avec le Java indonésien, mais aucun ne va jusqu'à admettre les idées novatrices de Tatsuo Hoshino, qui sont parfois même rejetées avec vigueur.

\footnotetext{
3 Shorto (1971) lwa' krom ja'ḅa' "Lawas, Cambodians and Laotians", Inscriptions of Burma, Pl. 358 26-7.

${ }^{4}$ Il existe de nombreuses publications sur la stèle de Ramkhamhèng. Le terme jvā est attesté à l'avantdernière ligne de la quatrième face. Une controverse, stérile à notre avis, veut que cette stèle soit un faux ; le problème est exposé avec objectivité par James R. Chamberlain (1991), The Ram Khamhaeng Controversy, collected papers edited by James R. Chamberlain.
} 
Nous allons voir que l'identité du Javā n'est qu'un élément des désignations des anciens Khmers par leurs voisins. Notre pérégrination sémantique partira de khmer ancien vnam (le Funan des chinois), se continuera par le javā de Jayavarman II et le ja'ba' des Môns, puis par le kur (ancien nom des Khmers) pour revenir au khmer moderne bhnam dont on tracera l'origine jusqu'aux premiers temps de l'agriculture. En conclusion, il sera montré que tous ces termes ne sont que des exonymes désignant les Khmers d'après leurs anciennes pratiques rituelles.

\section{Funan/Fou-nan (Fúnán 扶南)}

La mention d'un pays nommé Funan/Fou-nan (Fúnán 扶南) pour désigner la région qui deviendra le Cambodge apparaît pour la première fois dans l'Histoire des Trois Royaumes (Pelliot 1903) qui couvre la période 220-265. C'est, semble-t-il, George Cœdès qui a interprété Funan par le khmer moderne bhnam $\mathbf{p}^{\mathrm{h}}$ nom "montagne" (khmer ancien vnam bnəm ${ }^{5}$ très rarement bnam $)$. Le dictionnaire du Père Guesdon (1930: 1316) nous enseigne que à côté de "montagne", ce mot signifie aussi "monticule, tertre artificiel": bhnam khsāc' $\mathbf{p}^{\mathbf{h}}$ nom $\mathbf{k}^{\mathbf{h}} \mathbf{s a c}$ "monticules, cônes de sable artificiels (qu'on dresse comme œuvre méritoire)", bhnam yon $\mathbf{p}^{\mathbf{h}}$ nom jo:n "monticule, tertre, dressé pour crémation". Les recherches ethnographiques (Martin 1991 ; Porée-Maspero 1962-69) nous apprennent que les Khmers avaient l'habitude de pratiquer des cérémonies autour de tertres artificiels ; ces pratiques ont perduré jusqu'à l'époque récente. Par ailleurs, vnam est fréquemment attesté dans les inscriptions khmères du VIe au XIe siècles avec le sens de "temple" de n'importe quel type, petit temple ou temple-montagne. Cet usage n'a pas subsisté, et aujourd'hui les temples dénommés Phnom (Phnom Bakèng, Phnom Chisor) sont toujours situés au sommet d'une élévation naturelle. Il y a probablement eu pendant plusieurs siècles cohabitation des deux usages du terme: "tertre cérémoniel" dans l'usage populaire et "temple" dans l'usage des classes supérieures. C'est vraisemblablement dans le sens de "monticule, tertre artificiel (lieu de cérémonies)" plus que celui de "temple" et encore moins de "montagne" qu'il faut chercher la justification du nom Funan que les Chinois, frappés par des pratiques qu'ils ne connaissaient pas, ont donné au Cambodge des premiers siècles de notre ère.

Cette signification nous accompagnera tout au long de ce texte dans notre quête sur l'identification du terme Javā.

\section{Khmer javā et môn ja'ḅa'}

Je vais démontrer que le khmer javā correspond régulièrement au môn ja'ḅa' à l'aide de quelques correspondances phonétiques bien établies.

- Rappelons que la graphie $V$ en khmer ancien avait la valeur phonétique $b$ (aujourd'hui écrite $b$ et prononcée p). Exemples: ver/vyar "deux" (khmer moderne bïr pi:) ; vala “forces, soldat” du sanskrit bala (khmer mod. bal pol).

\footnotetext{
${ }^{5}$ Cette reconstruction résulte des travaux de William Baxter (1992) en phonétique historique du chinois. Voir aussi Ferlus (2005/2009).
} 
- Les préglottalisées initiales $* \boldsymbol{6}$ et $* \boldsymbol{d}$ du proto môn-khmer sont préservées en môn (écrites $b$ et $\underline{d}$ ) tandis qu'en khmer ancien elles se sont changées en occlusives sonores ordinaires $\mathbf{b}$ et $\mathbf{d}$ (écrites $v / b$ et $d$ ). Elles sont aujourd'hui prononcées $\mathrm{p}$ et $\mathrm{t}$ (écrites $b$ et $d$ ) à la suite du dévoisement de la série des occlusives initiales sonores au XVIIe siècle.

\begin{tabular}{|c|c|c|c|c|}
\hline Môn anc. & Môn moderne & Khmer ancien & \multicolumn{2}{|c|}{ Khmer moderne } \\
\hline$b \bar{a} r$ & 6a:] & ver/ber, vyar/byar & $b \bar{i} r[\mathrm{pi:}]$ & "deux" \\
\hline- & buih/kbuih [6oh] & - & buh [puh] & "bouillir" \\
\hline ḍāk, dẹk & $d \bar{a} k$ [daik] & $d i k$ & $d i ̣[\mathbf{t i k}]$ & “eau” \\
\hline dek, dik & $\operatorname{dik}$ [doik] & dik & - - - & "esclave" \\
\hline
\end{tabular}

- L'occlusive glottale finale 2 du proto môn-khmer est préservée en môn tandis qu'elle s'est effacée en khmer ancien en allongeant la voyelle.

\begin{tabular}{|c|c|c|c|}
\hline Môn anc. & Môn moderne & Khmer ancien & Khmer moderne \\
\hline$t i / t i$ & P] & ti/ & "terre" \\
\hline sro & sro $/ s w a '[$ sp?] & $s r \bar{u}$ & $s r \bar{u} V[$ sro:v] "paddy" \\
\hline
\end{tabular}

On peut donc pour le khmer ancien javā restituer la phonétique jəba: qui correspond régulièrement au môn ancien ja'ba' yə6a? (Shorto jə6 $\mathbf{a}^{2}$ ). Ces deux formes sont issues de proto môn-khmer *f.6a?. L'identification de la phonétique javā et sa correspondance avec le môn étant assurées, il n'y a plus de raison de situer le Javā de Jayavarman II en Indonésie.

Que pouvaient bien signifier ces termes? Contre toute attente, la réponse se trouve dans les langues du groupe aslien de la famille môn-khmer. Le kansiw du sud de la Thailande atteste caba " "montagne" (Phaiboon 2006). D'autres langues asliennes de Malaisie (semelai, banteg deq, chewong) attestent la même racine avec le sens de "colline, montagne" (Skeat \& Blagden 1906). Donc, le môn ancien ja'ḅa' fə6a? et le khmer ancien javā fəba: qui désignaient les (ou des) Khmers devaient avoir le sens premier de "tertre, monticule" comme bhnam et Funan.

\section{5. $J_{V \bar{a}}$ en thaï et en lao}

Les épigraphes thaï et les chroniques lao attestent la forme jvā dans l'ancien nom de Louang Phrabang (la translittération javā est possible car les graphies thaï et lao qui n'utilisent pas les souscrites ne permettent pas d'opposer les deux formes). La stèle dite de Ramkhamhèng qui relate les conquêtes de ce roi dans les quatre directions cite le Muang Chava (écrit jvā, lecture moderne $\mathbf{c}^{\mathbf{h}}$ awa:) "pays de Chava" comme le point le plus éloigné dans la direction du nord à partir de la capitale Sukhothay. Cette forme thaï n'est citée que dans les commentaires sur la stèle et est absente des dictionnaires modernes. La chronique historique de Louang Phrabang ${ }^{6}$ (Phinith 1987) atteste plusieurs fois jvā (lao २อๆ, prononcé swa:) pour l'époque légendaire (avant le XIVe

\footnotetext{
${ }^{6}$ Cette chronique, rédigée en 1870 à la demande du roi de Siam, a été élaborée d'après des manuscrits anciens qui ont tous été détruits lors de la mise à sac de Louang Phrabang en 1887 par les hordes de Đèo Văn Tri.
} 
siècle): mian் $j v \bar{a}$ "pays de Swa", miañ $j v \bar{a} ~ l a \bar{a} n^{2} j a \bar{a} \dot{n}^{2}$ "pays de Swa au million d'éléphants", Khūn jvā "roi de (Muang) Swa". Cette forme lao a été réactivée dans les usages modernes et est présente dans les dictionnaires.

Il faut bien insister sur le fait que la forme jvā est tirée de l'écrit, manifestement du khmer javā, et ne donne pas une idée de son ancienne prononciation. Cela pourrait indiquer que le ja'ba' des môns, continuation du Javā dont Jayavarman II avait rejeté la suzeraineté, n'était plus qu'un souvenir au début du XIVe siècle.

Si la prononciation de khmer javā n'a pas survécu dans les langues de la région, il est cependant possible que le môn ja'ḅa' se soit perpétué dans le nom de la liane Entada scandens (leguminosae) : thaï $\operatorname{saba:}^{\mathbf{c}^{1}}$ (สะบ้า) et lao $\mathrm{ba:}^{\mathbf{c}^{1}}$ (ข้ๆ), nommée en anglais "elephant creeper" ou "St Thomas bean". Cette liane donne une graine dure, plate et large (jusqu'à $5 \mathrm{~cm}$ ), qui est utilisée lors d'un jeu rituel dans l'aire môn et nyah kur (Diffloth 1984: 28) et qui rappelle notre 'jeu des osselets' (en anglais 'marbles game'). Si ce rapprochement est juste, le nom thaï de l'Entada scandens signifierait "le fruit de Javā".

\section{Kur}

Les Khmers sont actuellement nommés 'kur' par les Chams et les Bahnars. Contrairement à une idée courante, ce terme n'a rien à voir avec les attestations kmir et kvir (probablement une erreur de gravure) des inscriptions chames. Le terme 'kur' entre dans la composition de l'ethnonyme Nyah Kur qui désigne une population du nord-est de la Thaillande et dont la langue est proche parente du môn. Nyah Kur s'interprète par jàh "personne, les gens" (ex: Pajàh "qui") et kur "colline, montagne". Leur nom thaï 'Chao Bon' (ชาวบน), mot à mot "population des hauteurs", confirme bien le sens de 'kur'. Le môn moderne atteste ñaḥ jèh (môn ancien mñaḥ), mais 'kur' n'y est présent que par son emprunt birman kun.

La langue nyah kur comporte du vocabulaire d'origine khmère figé dans une phonétique du khmer moyen. Les Nyah Kur étaient inclus dans l'empire khmer et cet ethnonyme devait à cette époque désigner les Khmers dans la langue mône, ce qui expliquerait le sens restreint de kur "Khmer" en cham et en bahnar.

Ici encore, le sens premier de la désignation des Khmers est en rapport avec 'monticule, montagne'.

La racine 'kur' est peu répandue dans la région, en dehors des exemples cidessus on peut la trouver dans la langue des Por (groupe péarique) avec le sens de "bosse (cyphose)" (Brengues 1905).

\section{Synthèse linguistique et historique}

Que ce soit Funan (Fúnán 扶南) transcription chinoise du khmer ancien vnam bnəm (khmer moderne bhnam $\mathbf{p}^{\mathbf{h}}$ nom) pour désigner le premier pays khmer; que ce soit le khmer javā jəba: (le Javā de Jayavarman II) qui semble désigner le (ou une partie du) Zhenla de terre pour les autres Khmers, ou encore le môn ja'ḅa' fə6a? par lequel les anciens Môns devaient désigner ce même Zhenla; que ce soit enfin kur par lequel les Chams et les Bahnars d'aujourd'hui désignent les Khmers, et par lequel les 
anciens Môns ont dû à une certaine époque désigner les anciens Khmers du Nord-Est de la Thaïlande, tous ces vocables nous ramènent à la signification de "montagne" (au sens le plus large) et de "monticule, tertre" (au sens le plus restreint), précisons "tertre artificiel" comme lieu de culte et de cérémonies.

Il est donc bien acquis que les anciens Khmers, et eux seuls, étaient identifiés par leurs voisins comme le "(peuple des) monticules" parce qu'ils pratiquaient des cérémonies et des rites autour de monticules.

Que s'est-il donc passé au VIIIe siècle ? Pourquoi Jayavarman II unificateur des régions khmères ${ }^{7}$ libère-t-il son peuple du joug du Javā (forme khmère du môn ja'ba') - le Zhenla de terre - après l'avoir quitté, et pour finir se faire proclamer chakravartin "souverain universel" ? On sait que le nom de Zhenla remplace celui de Funan dans les sources chinoises à partir de 550. Si les limites et la composition du Funan sont très imprécises, le Zhenla quant à lui est proprement khmer, ou du moins à dominante khmère, et est considéré par les historiens comme le premier Cambodge. Au début du VIIIe, le Zhenla se scinde en deux entités distinctes : le Zhenla de terre au nord et le Zhenla d'eau au sud, la limite entre les deux se situant un peu plus bas que la frontière nord du Cambodge actuel. La spécification topographique ne fait qu'indiquer la prééminence des voies de communication, fluviale dans un cas ou terrestre dans l'autre.

La linguistique va nous aider à restituer la situation politique et à retracer la trame des événements. Au début du VIIIe siècle, le nord du Zhenla a dû tomber sous la domination des Môns qui l'ont nommé ja'ḅa' (traduction de bhnaṃ), transcrit javā par les Khmers. C'est donc la mainmise des Môns sur la moitié nord du Zhenla qui est la cause directe de la partition de ce pays en deux entités, le Zhenla de terre et le Zhenla d'eau. On connaît la suite, Jayavarman II, par conquêtes et reconquêtes, a réunifié le pays des Kambujas dont il s'est fait proclamer souverain universel en 802 sur le Phnom Kulen. Ces événements marquent le début de la prestigieuse période angkorienne.

On est beaucoup moins documenté sur ce qui s'est passé après cette date au nord de ce premier empire khmer issu de la réunification des deux Zhenla. Tatsuo Hoshino (1986) pense avec raison que le Javā (ou plutôt un lambeau de Javā) a survécu quelques temps dans la région de Louang Phrabang.

La répartition des Khamous (famille linguistique môn-khmer) au Nord-Laos montre qu'ils devaient constituer le fond de la population de la principauté de Louang Phrabang avant l'arrivée des Laos. Le mot khamou trla:s "marché" qui est à l'origine du lao tala:t (ถลๆด) a valeur de toponyme. Autre détail non moins intéressant, la langue khamou renferme quelques mots susceptibles d'être d'origine mône dont le plus significatif est ləgu:n “chef, important” (ləgu:n kuy "chef de village”; mar ləgu:n "python") qui correspond au môn moderne lagun ləkùn 'terme d'adresse honorifique pour les bonzes' (skt./pali guna “vertu, mérite"). ${ }^{8}$ L'usage de ləgu:n en khamou est l'indice d'une ancienne influence mône dans la région avant l'arrivée des

\footnotetext{
${ }^{7}$ C'est à dire des régions contrôlées par les Khmers. 'Khmer' ne doit pas être pris dans son sens ethnique restreint, à cette époque le Cambodge était largement pluri-ethnique.

${ }^{8}$ Ce terme n'est présent en thaï et en lao que par son emprunt direct au skt./pali.
} 
Laos. Par ailleurs, la présence des Môns est archéologiquement attestée dans la plaine de Vientiane où ont été mis au jour des sites môn des derniers siècles du premier millénaire (Gagneux 1972). Les toponymes en Vang (wăn อัง), du proto môn *way "circulaire" (môn moderne wan wèan "enclos, construction circulaire"), marquent l'ancien territoire môn depuis Vang Ka (wan் ka wèan ka?) au col des trois pagodes entre la Birmanie et la Thaïlande, jusqu'à Vang Vieng (wăn wien் อัๆอృ ) et Vang sang (wăn jāin $\dot{n}^{2}$ อัดฉ้ๆๆ) au nord de Vientiane.

Les données linguistiques corroborent assez bien la succession des événements historiques dans la région de Louang Phrabang : présence dominante d'une population de langue khamou (toponyme trla:s "marché"), suivie d'une période d'influence mône (titre ləgu:n “chef, important”), et pour finir laocisation de la région.

\section{Hypothèse sur l'origine de la désignation "(peuple des) monticules"}

Les Chinois ont nommé le pays des anciens Khmers Funan (Fúnán 扶南), transcription de vnam bnəm (khmer moderne bhnam $\mathbf{p}^{\mathbf{h}}$ nom) qui signifie à la fois "montagne" et "monticule, tertre cérémoniel". Les spécialistes n'ont retenu que le sens le plus majestueux en totale contradiction avec la topographie de plat pays qui est celle du premier territoire khmer au sud du Grand Lac. Or, les choix de vocabulaire dans les textes chinois sont toujours pertinents et c'est bien le sens de "tertre cérémoniel" qu'il convenait de retenir. C'est la langue khamou qui, encore une fois, vient à notre aide en confirmant cette interprétation.

Le khamou pnim "termitière", formation artificielle, correspond au khmer bhnam $\mathrm{p}^{\mathrm{h}}$ nom selon les lois régulières de la phonétique historique de ces langues. Par ailleurs, la rime -ìm est également attestée dans psìm "planter" et nim "année", ces deux mots étant en rapport de dérivation: "année" dérive de "planter" par le sens intermédiaire de "récolte", évolution étayée par le môn moderne cnāṃ ${ }^{\mathbf{h}}$ nam "année" dérivé infixal de cāṃ cam "repiquer les plants de riz" (Diffloth 1984: 134-135). Nous allons montrer que, en dépit du fossé sémantique apparent, pnìm "termitière" doit être rapproché de psìm "planter" et de nimm "année" dans une même famille de mots." Pour ce faire, quelques développements sur l'origine de l'agriculture vont être nécessaires.

Les botanistes spécialistes de l'histoire des plantes cultivées s'accordent aujourd'hui sur le fait que la première domestication de plantes alimentaires a eu lieu dans la zone tropicale humide de l'Asie du Sud-Est péninsulaire et insulaire. On sait également que la culture du riz — et du millet plus au nord — a été précédée par l'horticulture des tubercules, principalement le taro Colocasia et l'igname Dioscorea (Barrau 1974 ; Haudricourt et Hédin 1944 ; Sauer 1952). Le taro se cultive en champ humide comme le riz. Il y a d'ailleurs un lien historique entre ces deux plantes; il est probable que le riz a d'abord été une mauvaise herbe des fossés à taro. L'igname se cultive en champ sec et c'est ce tubercule qui va occuper notre attention.

\footnotetext{
${ }^{9}$ Cette strate de vocabulaire est trop ancienne pour permettre de restituer les règles morphologiques.
} 
L'igname est une liane à tubercule. Pour la cultiver, l'horticulteur doit affiner la terre et la préparer en monticules appelés billons (en anglais, earthing-up), où seront enfouies les semences (igname entier ou tronçonné en semenceaux). La liane viendra s'enrouler autour d'un tuteur fiché dans le sol. Nous ne savons rien des anciens horticulteurs mais il n'est pas hasardeux d'imaginer qu'ils pratiquaient des rites propitiatoires autour des billons pour assurer la fertilité et obtenir une bonne récolte de tubercules. Nous pensons, et c'est là notre théorie, que le bhnam $\mathbf{p}^{\mathbf{h}}$ nom des Khmers est le lointain avatar du billon d'igname. Le khamou pnim "termitière", tertre non naturel, devait à l'origine désigner le billon, ce qui expliquerait sa place dans une même famille de mots avec psìm "planter" et nìm "année". Les rites et cérémonies pratiqués autour des bhnam ne seraient en définitive que la survivance d'anciens rites agraires. Ces pratiques auraient été suffisamment caractéristiques pour que les Chinois des premiers siècles de notre ère désignent l'ancien pays des Khmers par Funan “(pays/peuple des) phnom”. Cette hypothèse, qui paraîtra sans doute audacieuse nous en acceptons les risques - repose sur trois types d'arguments: linguistique (existence d'un famille de mots regroupant les notions "planter", "année" et "billon"), cultural (ancienneté de l'horticulture des tubercules précédant l'agriculture céréalière du riz) et culturel (pratiques congénères de rites de fertilité).

\section{Du billon au phnom, et du phnom au temple-montagne ${ }^{10}$}

La linguistique nous a permis de restituer le fil conducteur qui va de 'billon' à 'phnom-(tertre)' et de ce dernier à 'phnom-(temple)', lequel évoluera vers l'emblématique temple-montagne.

Le temple-montagne héberge le linga (Stern 1934), plus précisément le linga royal dont le culte justifie le pouvoir du souverain. Le linga se présente comme un phallus, qu'il soit explicite sculpté avec précision ou suggéré dans une forme naturelle. L'essence du temple est dans la fonction du linga ou de son substitut, la statue de Shiva ; la magnificence de l'architecture et des décorations ne sont là que pour plaire aux divinités. Malgré les ruptures de civilisation que le Cambodge a connu, l'abandon des temples et l'oubli des rites associés, il est possible en se référant au modèle indien d'imaginer les pratiques autour du linga et de comprendre sa fonction. Mais, si le modèle indien a sa propre valeur dans le monde indien, ce modèle transposé en Asie du sud-est atteint ses limites et ne peut tout expliquer; il reste à déceler la part des traditions autochtones qui subsistent invisibles sous la massive magnificence indienne. Au Cambodge, le linga est au cœur du temple, il est ce par quoi le temple existe, et c'est dans la fonction du linga que se cache le secret de la permanence des rites khmers. Il est donc crucial de mettre au jour la signification khmère des pratiques autour du linga.

Dans l'Inde où les croyances n'ont pas connu de rupture, le linga n'est pas vénéré pour lui-même, mais en tant que signe de Shiva. Il est enchâssé dans un réceptacle ovoïde à bec considéré comme un symbole féminin, le yoni, complément

\footnotetext{
${ }^{10}$ Je remercie chaleureusement le professeur Bruno Dagens qui, avec beaucoup de patience, a bien voulu me faire profiter de sa grande connaissance des cultures de l'Inde.
} 
sacré du linga. Il n'est pas aisé d'obtenir des informations claires sur la fonction des pratiques entourant l'association linga-yoni. L'opinion la plus communément admise présente ces pratiques comme des rites propitiatoires de fécondité en général sans préciser s'il s'agit de fécondité humaine ou de fécondité agraire.

Au Cambodge, les explications proposées sont en général des reconstructions qui reposent, outre le modèle indien, sur l'archéologie et l'épigraphie. Le culte du linga y est devenu central, qu'il soit dressé dans un petit temple ou au sommet d'un templemontagne ( $c f$. fig. 1). Si sa représentation est explicite comme en Inde, la forme carrée $\mathrm{du}$ réceptacle ne suggère plus qu'une banale fonction de bassin pour recueillir l'eau d'ondoiement. Paradoxalement, ce sont les aménagements sur l'amont de deux ruisseaux affluents de la rivière de Siem Reap, les célèbres "rivières aux mille lingas" (Boulbet \& Dagens 1973) dans la région des Phnom Kulen, qui vont nous suggérer la signification des anciens cultes autour du linga bien mieux que ne saurait le faire un regard sur le modèle indien. En deux endroits, Kbal Spean (kpāl sbān) et Preah Thom (brah dham), les anciens Khmers ont gravé sur le socle rocheux de la rivière des centaines de petits linga schématisés par un carré avec une bosse ronde au centre (cf. fig. 2). Ici, la signification des cérémonies est écrite dans la topographie : l'eau courante, par ondoiement naturel, se charge de la semence divine virtuelle et s'écoule vers l'aval où elle ira fertiliser les rizières pour une bonne récolte. L'explication du processus est parfaitement transposable au linga du temple, du plus petit au templemontagne. Il est permis de penser que l'eau d'ondoiement évacuée par le bec du réceptacle était recueillie plus bas et utilisée pour fertiliser symboliquement les jardins et les rizières.

Les Khmers ne se sont pas contentés d'importer la culture indienne et ses représentations, ils l'ont interprétée et utilisée selon leurs besoins. ${ }^{11}$ Ces rites qui font dépendre la fertilité, et donc la prospérité de la population, du culte au linga assurent un pouvoir incontournable au souverain. Il est vraisemblable que c'est avec le culte du Devarāja, instauré par le brahmane savant de Jayavarman II, qu'a commencé cette association fusionnelle entre le culte du linga et le pouvoir royal qui est à l'origine de la somptueuse période angkorienne. En passant de l'Inde au Cambodge, l'image et le rôle du linga ont subi d'autres altérations. L'association linga-yoni s'est simplifiée pour devenir un linga dressé sur un bassin à bec destiné à recueillir l'eau d'ondoiement. On peut se demander au passage si ce n'était pas là justement sa fonction première en Inde! Je soupçonne les Indiens d'avoir élaboré une théorie intellectuelle - il ne peut en être autrement - en transformant un simple outils de culte populaire de fécondité en un symbole savamment élaboré de l'union des principes masculins et féminins incarnation de l'Univers. On a trop souvent oublié que les Indiens sont aussi de grands intellectuels et qu'ils sont tout aussi capables que les intellectuels occidentaux d'élaborer des théories savantes et embrouillées qui masquent la simplicité originelle des faits.

${ }^{11}$ Voir les développements de Bruno Dagens (2009). 


\section{Brèves conclusions}

En définitive, le culte du linga sur le temple-montagne dans sa version la plus majestueuse ne serait que l'aboutissement en droite ligne de rites agraires élémentaires accomplis par les horticulteurs pré-néolithiques autour des billons d'ignames, perpétués en suivant un long cheminement dans le temps qui conduit du billon au phnom-(tertre), puis au phnom-(temple) pour s'achever au temple-montagne. C'est un exemple édifiant de la remarquable permanence des rites agraires primordiaux, franchissant les millénaires malgré les grands changements historiques et culturels, et toujours vivants sous la chape de la culture indienne.

Cette étude permet en outre de remettre à l'honneur l'hypothèse de l'historien Tatsuo Hoshino sur la localisation du Javā et son identification avec le Zhenla de terre. Grâce à la linguistique, on a montré comment les anciens Khmers étaient connus comme "(le peuple des) tertres cérémoniels" sous les différentes désignations analysées plus haut : khmer ancien vnam (moderne bhnam $\mathbf{p}^{\mathrm{h}}$ nom) et sa transcription chinoise Funan, môn ja'ḅa' et sa transcription khmère javā, et enfin Bahnar et Cham kur.

Une fois de plus, il est montré comment un bon usage des données linguistiques peut aider à apporter une réponse aux interrogations des historiens sur certains problèmes ponctuels. Cette démarche confirme par dessus tout la nécessité d'une approche pluridisciplinaire comme l'a toujours préconisé le regretté André G. Haudricourt. ${ }^{12}$

Loin de clore le débat, cette étude devrait contribuer à renouveler la réflexion sur certains point d'histoire chez les historiens de l'Asie du Sud-Est et du Cambodge et en particulier, revoir la fonction des temples et des rites associés à la lumière des rites agraires des anciens Khmer autour du phnom des origines.

\footnotetext{
${ }^{12}$ Pour un aperçu sur la pensé et l'œuvre de André G. Haudricourt on peut se reporter à son passionnant ouvrage Les pieds sur terre (1987), écrit en collaboration avec Pascal Dibie.
} 


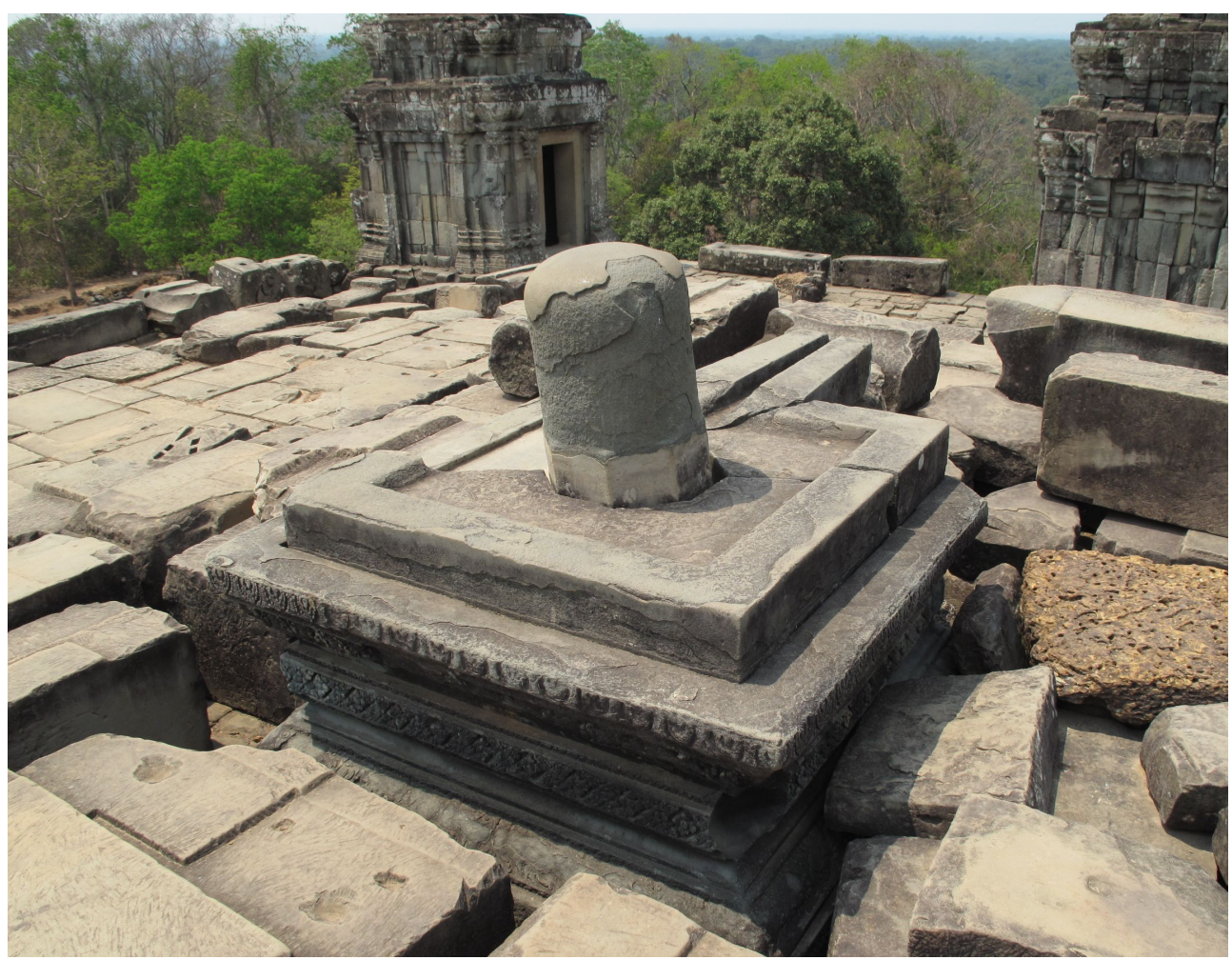

Image 1 : linga sur temple (Phnom Bakèng)

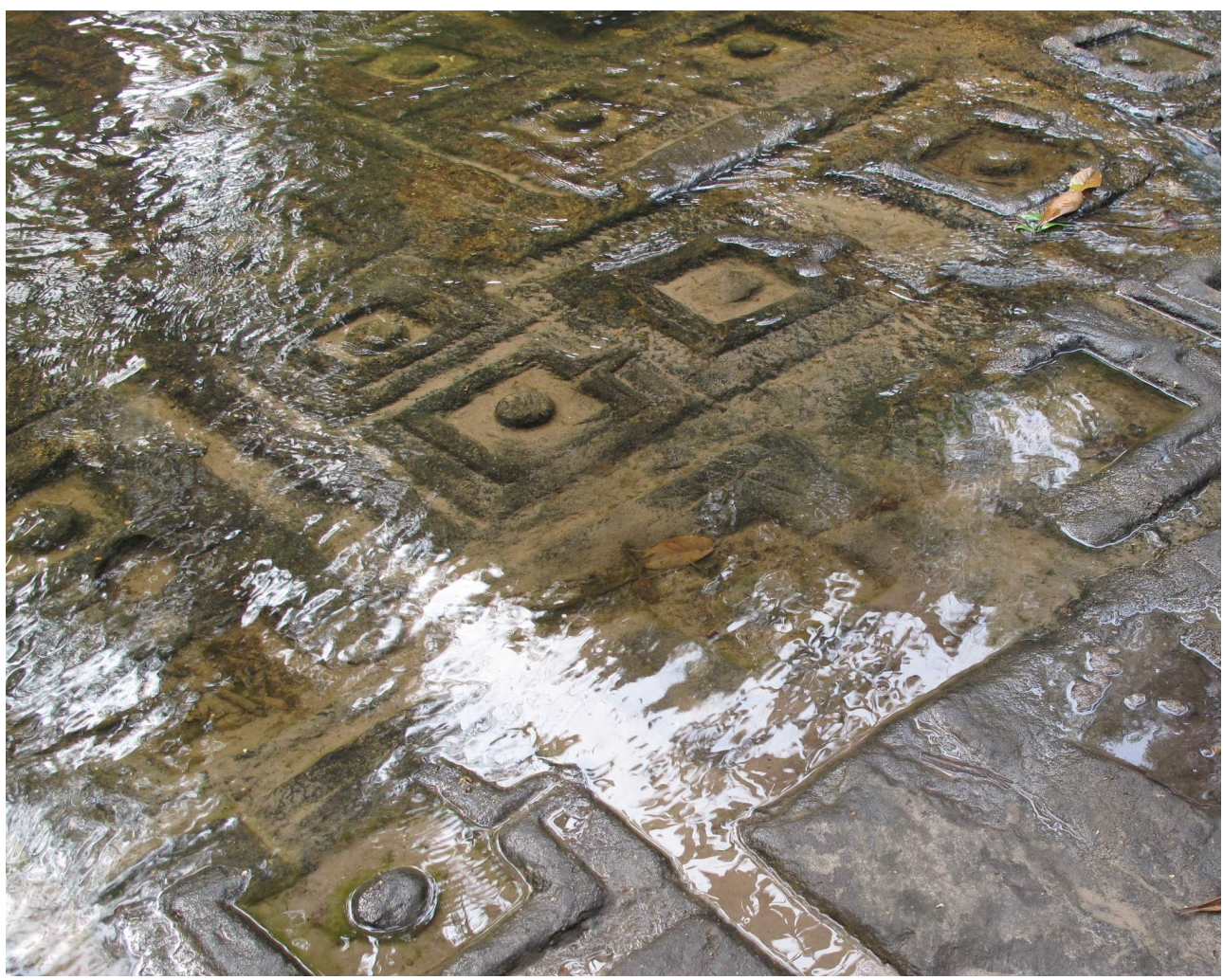

Image 2 : linga sur fond rocheux de la rivière (Kbal Spean) 


\section{Références}

Aymonier, Etienne. 1901. La stèle de Sdok Kâk Thom, Journal Asiatique, 9e série, 17: 5-52.

BARraU, Jacques. 1974. L'Asie du Sud-Est, berceau cultural, Etudes Rurales 53-56: 18-40, Paris/La Haye, Mouton \& Co.

BaXter, William H. 1992. A Handbook of Old Chinese Phonology, Berlin/New York, Mouton de Gruyter.

Boulbet, Jean \& Bruno DAgens. 1973. Les sites archéologiques de la région du Bhnam Gūlen (Phnom Kulen), Arts Asiatiques XXVII, Paris, Annales du Musée Guimet et du Musée Cernuschi.

BREngues, Docteur Jean. 1905. Notes sur les populations de la région des montagnes des Cardamomes, The Journal of the Siam Society 2(1): 19-47.

Chamberlain, James R. (ed.). 1991. The Ramkhamhaeng Controversy, Bangkok, The Siam Society.

Coedes, George. 1948. Les États hindouisés d'Indochine et d'Indonésie, Paris, De Boccard. Réimpression 1989.

CoEdES, George \& Pierre DuponT. 1943-46. La stèle de Sdok Kak Thom, BEFEO 43: 56-134.

Dagens, Bruno. 2003. Les Khmers, Paris, Les Belles Lettres.

DAGENS, Bruno. 2009. Mythologie indienne et pouvoir royal dans le Monde Indien: l'exemple du Cambodge ancien, Mythes et mythologies (Actes du Colloque international des 6, 7 et 8 mars 2008 à Amiens), Amiens, Presses du "Centre d'Études Médiévales", Université de Picardie.

Diffloth, Gérard. 1984. The Dvaravati Old Mon Language and Nyah Kur, Bangkok, Chulalongkorn University Printing House.

FERLus, Michel. 2005. L'intérêt linguistique des transcriptions chinoises concernant le Cambodge ancien (Fou-nan et Tchen-la), Dix-neuvièmes journées de linguistique de l'Asie Orientale, CRLAO (EHESS-CNRS), 30 juin- $1^{\text {er }}$ juillet 2005. "id. » 2009, Bulletin de l'AEFEK n ${ }^{\circ} 15$. http://aefek.free.fr/pageLibre00010944.html

FINOT, Louis. 1903. Note d'épigraphie V, Paduranga, BEFEO 3: 630-648.

FinOT, Louis. 1915. Notes d'épigraphie XVI, L'inscription de Sdok Kak Thom, BEFEO 15(2): 53-106.

GagneuX, Pierre Marie. 1972. Vers une révolution dans l'archéologie indochinoise, Bulletin des amis du Royaume Lao 7-8: 83-105.

GondA, Jan. 1973. Sanskrit in Indonesia, New Delhi, Aditya Prakashan. Reprint 1998.

GuESDON, Père Joseph. 1930. Dictionnaire Cambodgien-Français, 2 vols., Paris, Plon.

Haudricourt André G. \& Louis Hedin. 1944. L'Homme et les plantes cultivées, Paris, Gallimard. Réédition 1987, Paris, A.M. Métaillié.

HAudricourt André-Georges \& Pascal Dibie. 1987. Les pieds sur terre, Paris, A.M. Métaillié.

Hoshino, Tatsuo. 1986. Pour une histoire médiévale du Moyen Mékong, Bangkok, Editions Douang Kamon. [Publication d'un mémoire de l'EHESS, 1976].

Hoshino, Tatsuo. 2002. Wen Dan and its Neighbours: The Central Mekong Valley in the Seventh and Eighth Century, Breaking New Ground in Lao History, ed. by Mayoury Ngaosrivathana \& Kennon Breazeale, Bangkok, Silkworm Books. 
JACQUES, Claude. 1972. Études d'épigraphie cambodgienne VIII, La carrière de Jayavarman II, BEFEO 59: 205-220.

JACQUES, Claude. 2005. Deux problèmes posés par la stèle de Prah Khan K. 908, Aséanie 15: 11-31.

Jenner, Phillip N. 2009a. A dictionary of pre-Angkorian Khmer, Doug Cooper ed., Pacific Linguistics 597, Australian National University.

Jenner, Phillip N. 2009b. A dictionary of Angkorian Khmer, Doug Cooper ed., Pacific Linguistics 597, Australian National University.

KuRZ, Johannes L. 2006. Boni in Chinese Sources: Translations of Relevant Texts from the Song to the Qing Dynasties ».

Long SEAm. 2000. Dictionnaire $d u$ khmer ancien (d'après les inscriptions $d u$ Cambodge du VIe.-VIIIe. siècles), Fondation Toyota du Japon, Phnom Penh Printing House.

MARTIN, Marie. 1991. Le phnom yong, monument funéraire de l'ouest cambodgien, Cahiers de l'Asie du Sud-Est 29-30: 297-308.

Pelliot, Paul. 1903. Le Fou-nan, BEFEO 3(2): 248-303.

Pelliot, Paul. 1904. Deux itinéraires de Chine en Inde à la fin du VIIIe siècle, BEFEO 4(1-2): 131-413.

Phaiboon Duang Chan. 2006. The northern Aslian languages of southern Thailand, MKS 36: 207-224.

Phinith, Saveng. 1987. Contribution à l'histoire du Royaume de Luang Prabang, Paris, École Française d'Extrême-Orient.

Poree-Maspero, Éveline. 1962. Études sur les rites agraires des Cambodgiens, 3 vols., Paris, Mouton.

Pou, Saveros. 1992. Dictionnaire vieux khmer-français-anglais, Cedoreck, Paris. Nouvelle édition augmentée d'un supplément, 2004, Paris, L'Harmattan.

Renou, Louis \& Jean Filliozat. 1985. L'Inde classique, tome I, Paris, Adrien Maisonneuve.

Sak Humphry, ChHAny. 2001. The Sdok Kak Thom Inscription (K.235), [Phnom Penh], The Edition of the Buddhist Institute.

SAUER, Carl O. 1952. Agricultural Origins and Dispersals, New York, American Geographical Society, George Grady Press.

SHORTo, Harry L. 1971. A Dictionary of the Mon Inscriptions from the Sixth to the Sixteenth Centuries, London, Oxford University Press.

Skeat, Walter W. \& Blagden, Charles Otto. 1906. Pagan Races of the Malay Peninsula, 2 vol., London, Frank Cass. Reprint 1966.

STERN, Philippe. 1934. Le temple-montagne khmèr, le culte du linga et le devarāja, BEFEO 34: 611-616.

VICKerY, Michael. 1998. Society, Economics, and Politics in Pre-Angkor Cambodia, The Center for East Asian Cultural Studies for Unesco.

Wolters, Olivier W. 1973. Jayavarman II's Military Power: The Territorial Foundation of the Angkor Empire, JRAS pt. 1: 21-30. 